PTU-57 DIRECT AND NON-DIRECT COSTS IN DIRECT-TO-TEST UPPER GASTROINTESTINAL ENDOSCOPY: NURSE ENDOSCOPISTS VERSUS DOCTORS

${ }^{1}$ Ahaan Gupta*, ${ }^{2} J a c o b o$ Ortiz-Fernandez-Sordo. 'University of Nottingham Medical School, UK, ${ }^{2}$, Nottingham University Hospitals NHS Trust, UK

\subsection{6/gutjnl-2021-BSG.130}

Introduction Nurses can perform endoscopic procedures with safety and efficacy, however, there is no definitive evidence surrounding their cost-effectiveness. Our aim was to perform a cost analysis of direct-to-test oesophagogastroduodenoscopy (DTT OGD) in Nottingham University Hospitals NHS trust by comparing direct, non-direct, and total costs between nurse endoscopists and doctors. It was hypothesised that nurses will be more costly despite their lower salaries due to the higher subsequent episodes of care their patients are likely to require. Methods This was a longitudinal retrospective observational study in the form of a clinical audit. Data was collected and analysed for 808 patients referred from primary care for a DTT OGD performed by nurse endoscopists $(n=338)$ and doctors $(n=470)$ from January 2016 to April 2017. Direct cost variables included costs incurred during the DTT OGD procedures such as the salaries of endoscopists and assistants, consumables, pre-medication, sedation, histopathological analysis of biopsy pots, rapid urease tests and prescriptions. Nondirect cost variables involved costs of the subsequent episodes of care arranged until one year as a result of the DTT OGD such as additional diagnostic tests, repeat/follow-up OGDs, specialist clinic appointments and upper gastrointestinal multidisciplinary team meetings. Due to the abnormal distribution of costs, it was not possible to compare the mean costs between the two groups of endoscopists using the Independent Samples t-test. Hence, the Mann-Whitney U test on Statistical Package for Social Sciences was used for data analysis.

Results Doctors compared to nurses had a lower median direct cost $(£ 130.98$ vs. $£ 197.56, \mathrm{p}=0.948)$ and lower median total cost ( $£ 131.43$ vs. $£ 197.56, \mathrm{p}=0.315)$. Direct costs were higher for nurses due to a higher number of biopsies taken for histopathological analysis, but statistically significant differences were not observed. The median non-direct cost ( $£ 0$ vs. $£ 0$, $\mathrm{p}=0.494$ ) was the same for both groups because most patients had no subsequent follow-ups arranged. However, nurses had a higher mean non-direct cost (£48.66 vs. $£ 69.34)$ as they arranged more repeat/follow-up OGDs.

Conclusions Lower direct, non-direct and total costs of performing DTT OGD were observed in the doctor group compared to the nurse endoscopist group. This might indicate a difference between doctors and nurses in their confidence, clinical decision-making and non-technical skills. However, differences in costs were not statistically significant between the two groups. Hence, the distribution of costs was the same across nurse endoscopists and doctors. Based on these findings, further large-scale research in the form of economic evaluations is warranted to determine whether nurse endoscopists are cost-effective in performing endoscopies.

\section{PTU-58 METAL VERSUS PLASTIC STENTING IN MALIGNANT BILIARY OBSTRUCTION}

Ben Arnold, Rachel Perry*, Ben Colleypriest. Royal United Hospital, Bath, UK
Introduction Decompression by ERCP and stenting is often the first-line management in cases of malignant biliary obstruction. Not infrequently this is performed to relieve symptoms prior to tissue diagnosis and definitive management. For this reason removable stents are favoured pending tissue diagnosis and surgical assessment for resectability. When necessary the ESGE 2017 guideline recommends the use of self-expanding metal stents (SEMS) over plastic stents in pre-operative biliary drainage (PBD) and fully covered SEMS (fcSEMS) when the aetiology of biliary obstruction is unclear. PBD with SEMS has been shown to lower requirement for endoscopic re-intervention without affecting surgical outcomes ${ }^{1}$ when compared to plastic stents.

Methods Retrospective data from January 2017 - April 2020 were collected from our endoscopy database to identify all procedures in which either metal (SEMS and fcSEMS) or straight plastic stent were deployed. Electronic hospital records (including clinic letters, MDT outcomes, imaging and blood results) were accessed to identify the indication and potential complications of post-ERCP pancreatitis (PEP), cholangitis, cholecystitis, stent occlusion and stent migration.

Results 163 procedures (54 fcSEMS; 36 PS; 73 SEMS) were identified. Of these 42 of fcSEMS, 26 in PS and 64 of SEMS were indicated for HPB malignant obstruction. Pancreatic cancer was by far the most common indication. The remainder of procedures were for benign disease or other cancers.

Overall PEP rates were 14.8\%, 11.4\% and $0 \%$ for fcSEMS, PS and SEMS respectively. When benign and non-HPB primary cases were excluded, the frequency of PEP was higher in fcSEMS (16.6\%) compared to PS (15.4\%) although not statistically significant $(\mathrm{p}=0.89)$. The highest PEP rate $18.4 \%$ was observed in fcSEMS with preceding sphincterotomy. Our baseline PEP rate was $2.85 \%$ for the same period $(95 \% \mathrm{CI}$, 1.82-4.25).

Occlusion rate was $11.9 \%$ at a (mean126 days) in fcSEMS, $23 \%$ (mean 60 days) in PS and $11.3 \%$ in SEMS (mean 83 days ) although, again, this difference is not statistically significant $(p=0.26)$. Cholangitis was seen in $9.6 \%$ of fcSEMS vs $7.7 \%$ PS group vs $6.3 \%$ SEMS group. We identified no cases of procedure related cholecystitis in our data. Migration rate of stents was 7.1\%, $0 \%$ and $1.6 \%$ in fcSEMS, PS and SEMS groups respectively.

$13 \mathrm{fcSEMS}$ patients eventually proceeded to attempted surgery, $8(19 \%)$ had resectable disease at time of surgery. All SEMS patients were stented with palliative intent. 5 PS patients went for surgery and $311.5 \%$ underwent curative resection.

Conclusions While our data did not yield statistically significant results, it does highlight the high complication rate of both fcSEMS and PS in biliary decompression. PEP rates were significantly higher than baseline and sphincterotomy prior insertion of fcSEMS seems to increase this risk further. Stent occlusion occurred more frequently and sooner in PS but at a mean of 60 days, this would often prove long enough to confirm diagnosis and plan definitive treatment. Plastic stents are preferred over metal prior to EUS staging and the majority of patients will go on require this in their work up.

\section{REFERENCE}

1. Crippa $S$, Cirocchi $R$, Partelli $S$ et al. Systematic review and metaanalysis of metal versus plastic stents for preoperative biliary drainage in resectable periampullary or pancreatic head tumors. Eur J 